Xiaofeng Yang and Baowei Fei, "A skull segmentation method for brain MR images based on multiscale bilateral filtering scheme", Benoit M. Dawant, David R. Haynor, Editors, Proc. SPIE 7623, 76233K (2010)

Copyright 2010 Society of Photo-Optical Instrumentation Engineers (SPIE). One print or electronic copy may be made for personal use only. Systematic reproduction and distribution, duplication of any material in this paper for a fee or for commercial purposes, or modification of the content of the paper are prohibited.

http://dx.doi.org/10.1117/12.844677 


\title{
A Skull Segmentation Method for Brain MR Images Based on Multiscale Bilateral Filtering Scheme
}

\author{
Xiaofeng Yang and Baowei Fei * \\ Departments of Radiology, Emory University, Atlanta, GA \\ * Corresponding Author: bfei@emory.edu
}

\begin{abstract}
We present a novel automatic segmentation method for the skull on brain MR images for attenuation correction in combined PET/MRI applications. Our method transforms T1-weighted MR images to the Radon domain and then detects the feature of the skull. In the Radon domain we use a bilateral filter to construct a multiscale images series. For the repeated convolution we increase the spatial smoothing at each scale and make the cumulative width of the spatial and range Gaussian doubled at each scale. Two filters with different kernels along the vertical direction are applied along the scales from the coarse to fine levels. The results from a coarse scale give a mask for the next fine scale and supervise the segmentation in the next fine scale. The method is robust for noise MR images because of its multiscale bilateral filtering scheme. After combining the two filtered sinogram, the reciprocal binary sinogram of the skull is obtained for the reconstruction of the skull image. We use the filtered back projection method to reconstruct the segmented skull image. We define six metrics to evaluate our segmentation method. The method has been tested with brain phantom data, simulated brain data, and real MRI data. Evaluation results showed that our method is robust and accurate, which is useful for skull segmentation and subsequently for attenuation correction in combined PET/MRI applications.
\end{abstract}

Keywords: skull segmentation, Radon transform, bilateral filter, multiscale scheme, PET/MRI

\section{INTRODUCTION}

The segmentation of the skull in medical images is an important step toward complete segmentation of the tissue in the human head, the latter being an indispensable step in the construction of a realistic model of the head. Attenuation correction (AC) is an essential step in the study of quantitative PET imaging of the brain. However, the current design of Siemens combined PET/MRI does not offer transmission scans for attenuation correction because of space limit. One of approaches [1] is to use MR images for attenuation correction of PET images. By segmenting the brain into the scalp, skull, and brain tissue, one can assign different attenuation coefficients to the segmented tissue and then produce the attenuation correction map. On the AC map, the skull has the largest attenuation coefficient; and its effect dominates the whole attenuation correction. Once the skull is segmented from MR images, the scalp that is outside of the skull, and the brain tissue that is inside of the skull, can be obtained. Therefore, skull segmentation is a key step for MRI-based attenuation correction.

A number of techniques have been proposed to automate the segmentation of brain structures in MR images ([2-4]) Subsequent work focused on developing automated algorithms specifically for brain MR images. A recent study published by Segonne [2] divided current automatic approaches for skull stripping into three categories: region based, boundary based and hybrid approaches. In [3], Zhuang categorized skull stripping methods into three types: intensity based, morphology based and deformable model based. Techniques have also been developed that make use of information from multiple modalities to segment the brain ([4] and [5,6]). A recent study published by Fennema et al.[7] compared the performance of the most commonly used skull stripping algorithms. This study concluded that existing algorithms had both strengths and weaknesses, but that no single algorithm was robust enough for large scale analyses. This finding was in agreement with other recently published studies ([8] and [9]). Due to the presence of imaging

Medical Imaging 2010: Image Processing, edited by Benoit M. Dawant, David R. Haynor,

Proc. of SPIE Vol. 7623, 76233K - (C) 2010 SPIE - CCC code: 1605-7422/10/\$18 - doi: 10.1117/12.844677

Proc. of SPIE Vol. 7623 76233K-1 
artifacts, anatomical variability, varying contrast properties, and poor registration, most of these techniques do not give satisfactory results over a wide range of scan types and neuroanatomy without manual intervention.

In this paper, we propose a novel automatic segmentation method for the skull on brain MR images for attenuation correction in combined PET/MRI applications. Our method transforms T1-weighted MR images to the Radon domain and then detects the feature of the skull. In the Radon domain we use a bilateral filter to process MR brain images and to construct a multiscale images series. For the repeated convolution we increase the spatial smoothing at each scale and make the cumulative width of the spatial and rang Gaussian doubled at each scale. Two filters with different kernels along the vertical direction are applied along the scales from the coarse to fine levels. The result from a coarse scale supervises the segmentation in the next fine scale. After combining the two filtered sinogram, the results from a coarse scale give a mask for the next fine scale and supervise the segmentation. The method has been tested with brain phantom data, simulated brain data, and real MRI data. In the next sections, we will report the methods and evaluation results.

\section{METHODS}

\subsection{Radon transform}

As described in Figure 1, our Radon transform is defined as follows, i.e. from a complete set of line integrals $P_{s}(\alpha)$.

$$
P_{s}(\alpha)=R f(\alpha, s)=\int_{-\infty}^{\infty} \int_{-\infty}^{\infty} f(x, y) \delta(s-x \cos \alpha-y \sin \alpha) d x d y \quad \alpha \in[0, \pi] \quad s \in R
$$

Where $s$ is the perpendicular distance of a line from the origin and $\alpha$ is the angle formed by the distance vector. From this figure we can see the projection image, i.e. 'sinogram' will has two gaps because of low signal of the skull. The point $f(x, y)$ on the brain image is corresponding to the sine curve in the Radon domain. According to the Fourier slice theorem, this transformation is invertible. For a 2-D function $f(x, y)$, the 1-D Fourier transforms of the Radon transform along $S$, are the 1-D radial samples of the 2-D Fourier transform of $f(x, y)$ at the corresponding angles.

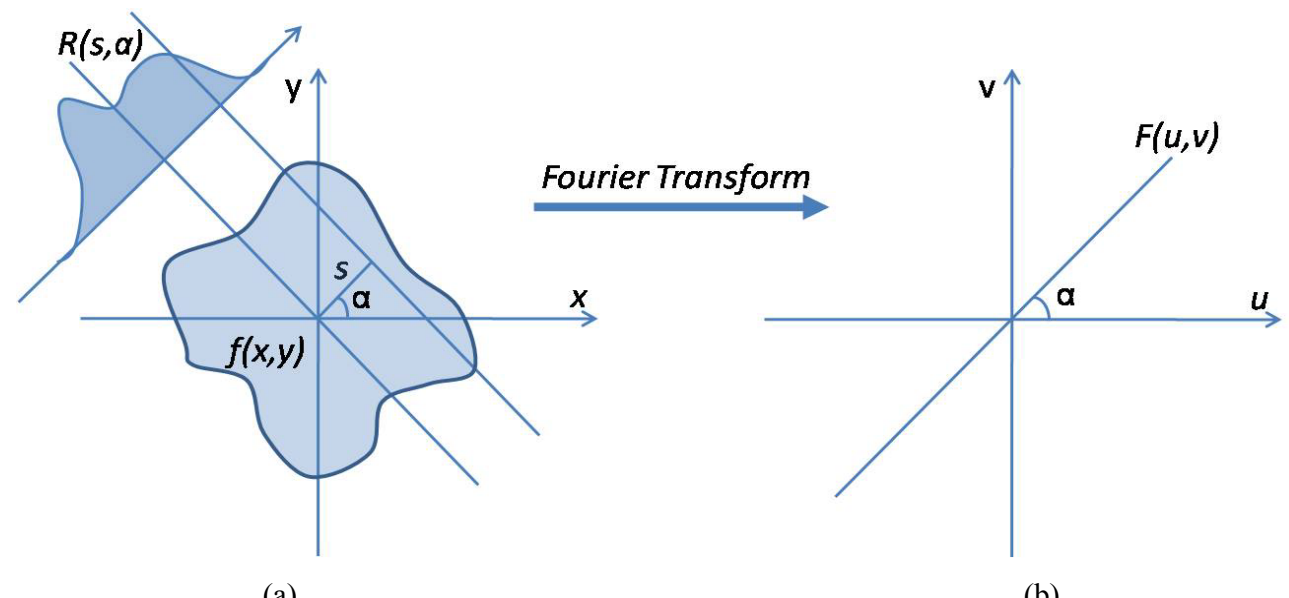

(a)

(b)

Figure 1. The schematic diagram of Radon Transformation. (a) Radon transform of an image. (b) 1D Fourier transforms of the projections, which make the $2 \mathrm{D}$ Fourier transform of the image.

After the Radon transformation, the MR image is transformed from the image domain to the Radon domain. Figure 2 shows the MR image and the corresponding sinogram in the Radon domain. From the sinogram on Figure 2, we can see that there are two low intensity gaps on the top and bottom sides of the sinogram along the vertical direction, which indicates the skull that has relatively low signal intensity on the T1-weighted MR image. 


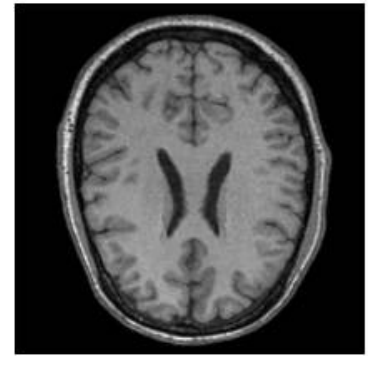

(a)

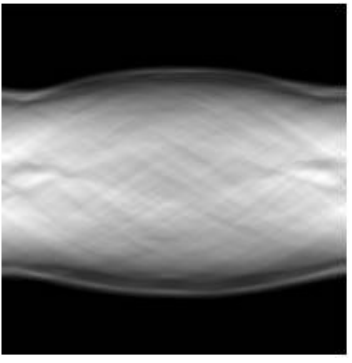

(b)

Figure 2. MR image and corresponding sinogram. (a) T1-weighted MR brain image. (b) The corresponding sinogram.

In Figure 3 (a) we added $5 \%$ and $15 \%$ into the original MR image. The image with $15 \%$ noise has already been contaminated severely and we cannot see the details. In Figure 3 (b) the profiles drawn through the corresponding sinogram is very close, and it shows that our transform has greatly decreased the noise in the original image.

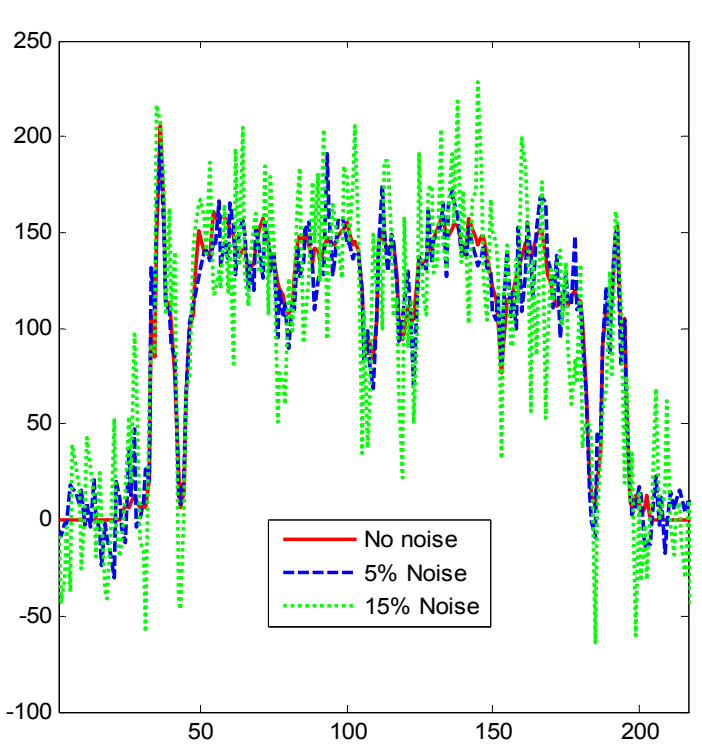

(a)

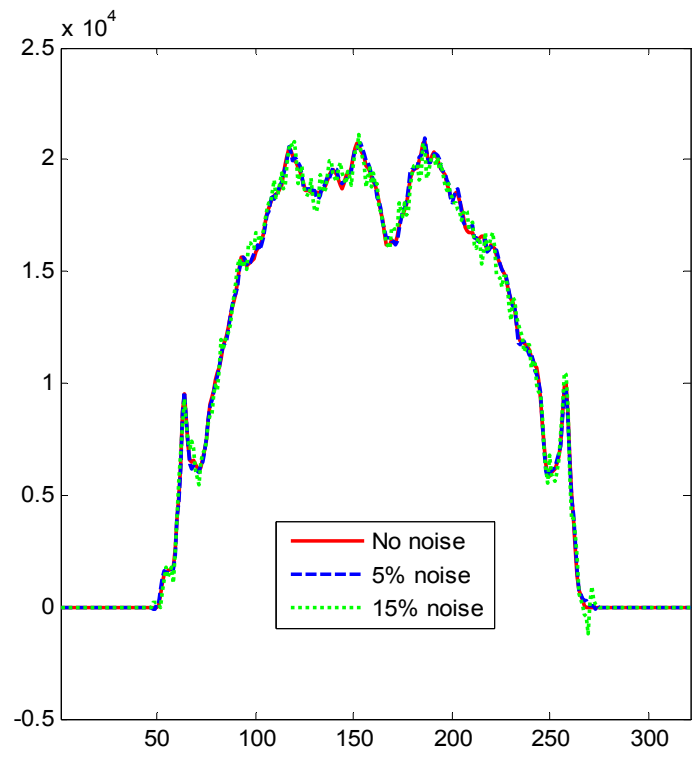

(b)

Figure 3. Comparison of line profiles. (a) The profiles between original and noised MR images. (b) The profiles between the corresponding sinograms.

\subsection{Multiscale space from bilateral filtering}

The multiscale space represents a series of images with different levels of spatial resolution. General information is extracted and maintained in images at the coarse scale, and images at the fine scale have more local tissue information. Bilateral filtering can introduce a partial edge detection step into the filtering process so as to encourage intra-region smoothing and preserve the inter-region edge.

\subsubsection{Bilateral filtering}

Bilateral filtering is a non-linear filtering technique introduced by Tomasi et al [10]. This filter is a weighted average of local neighborhood samples, where the weights are computed based on temporal (or spatial in case on images) and 
radiometric distance between the center sample and the neighboring samples. It smoothes images while preserving edges, by means of a nonlinear combination of nearby image values. Bilateral filtering can be described as follows:

$$
h(x)=\lambda^{-1}(x) \int_{-\infty}^{\infty} \int_{-\infty}^{\infty} I(\xi) W_{\sigma_{s}}(\xi-x) W_{\sigma_{r}}(I(\xi)-I(x)) d \xi
$$

with the normalization that ensures that the weights for all the pixels add up to one.

$$
\lambda(x)=\int_{-\infty}^{\infty} \int_{-\infty}^{\infty} W_{\sigma_{s}}(\xi-x) W_{\sigma_{r}}(I(\xi)-I(x)) d \xi
$$

Where $I(x)$ and $h(x)$ denote the input and output images, respectively. $W_{\sigma_{s}}$ measures the geometric closeness between the neighborhood center $x$ and a nearby point $\xi$, and $W_{\sigma_{r}}$ measures the photometric similarity between the pixel at the neighborhood center $x$ and that of a nearby point $\xi$. Thus, the similarity function $W_{\sigma_{r}}$ operates in the range of the image function $I$, while the closeness function $W_{\sigma_{s}}$ operates in the domain of $I$.

Bilateral filter replaces the pixel value at $x$ with an average of similar and nearby pixel values. In smooth regions, pixel values in a small neighborhood are similar to each other, and the bilateral filter acts essentially as a standard domain filter, averaging away the small, weakly correlated differences between pixel values caused by noise.

\subsubsection{Gaussian kernel}

Many kernels can be used in bilateral filtering. A simple and important case of bilateral filtering is shift-invariant Gaussian filtering, in which both the spatial function $W_{\sigma_{s}}$ and the range function $W_{\sigma_{r}}$ are Gaussian functions of the Euclidean distance between their arguments. More specifically, $W_{\sigma_{s}}$ is described as:

$$
W_{\sigma_{s}}(\xi-x)=e^{-\frac{1}{2}\left[d_{s}^{2} / \sigma_{s}^{2}\right]}
$$

where $d_{s}=\|\xi-x\|$ is the Euclidean distance. The range function $W_{\sigma_{r}}$ is perfectly analogous to $W_{\sigma_{s}}$ :

$$
W_{\sigma_{r}}(\xi-x)=e^{-\frac{1}{2}\left[d_{r}^{2} / \sigma_{r}^{2}\right]}
$$

where $d_{r}=|I(\xi)-I(x)|$ is a suitable measure of distance in the intensity space. In the scalar case, this may be simply the absolute difference of the pixels. Just as this form of domain filtering is shift-invariant, the Gaussian range filter introduced above is insensitive to overall additive changes of image intensity. Thus, the range filter is shift-invariant as well.

\subsubsection{Multiscale bilateral decomposition}

Bilateral filtering is a scale space, and multiscale bilateral decomposition technique which smoothes the images as the scale increases. When the scale increases, the images become more blurred and contain more general information. For an input discrete image $I$ the goal of the multiscale bilateral decomposition [11] is to first build a series of filtered images $I^{i}$ that preserve the strongest edges in $I$ while smoothing small changes in intensity. The original image is the $0^{\text {th }}$ scale ( $i=0$ ), then iteratively apply the bilateral filter to compute 
with

$$
\begin{aligned}
I_{n}^{i+1} & =\frac{1}{\lambda} \sum_{k \in \Omega} W_{\sigma_{s}, i}(k) \cdot W_{\sigma_{r}, i}\left(I_{n+k}^{i}-I_{n}^{i}\right) \cdot I_{n+k}^{i} \\
\lambda & =\sum_{k \in \Omega} W_{\sigma_{s}, i}(k) \cdot W_{\sigma_{r}, i}\left(I_{n+k}^{i}-I_{n}^{i}\right)
\end{aligned}
$$

where $n$ is a pixel coordinate, $W_{\sigma}(x)=\exp \left(-x^{2} / \sigma^{2}\right), \sigma_{s, i}$ and $\sigma_{r, i}$ are the widths of the spatial and range Gaussians, respectively; and $k$ is an offset relative to $n$ that runs across the support of the spatial Gaussian. The repeated convolution by $W_{\sigma_{s}, i}$ increases the spatial smoothing at each scale $i$, and we choose the $\sigma_{s, i}$ so that the cumulative width of the spatial Gaussian doubles at each scale. Suppose that at the finest scale we set the spatial kernel $\sigma_{s, 1}=\sigma_{s}$. Then setting $\sigma_{s, i}=2^{i-1} \sigma_{s, i-1}$ for all $i>1$, we obtain a width of $\left(2^{i}-1\right) \sigma_{s}$ for the cumulative spatial Gaussian.

The range Gaussian $W_{\sigma_{r}, i}$ is an edge-stopping function. Ideally, if an edge is strong enough to survive after several iterations of the bilateral decomposition, we would like to preserve it. To ensure this property we set $\sigma_{r, i}=2^{i-1} \sigma_{r}$. Increasing the width of the range Gaussian by a factor of 2 at every scale increases the chance that an unwanted edge that survives at previous iteration will be smoothed away in later iterations.

Figure 4 illustrates the scale space which was constructed using multiscale bilateral filtering. Unlike many multiresolution techniques where the images are down-sampled along the resolution, we kept the image resolution along the scales.

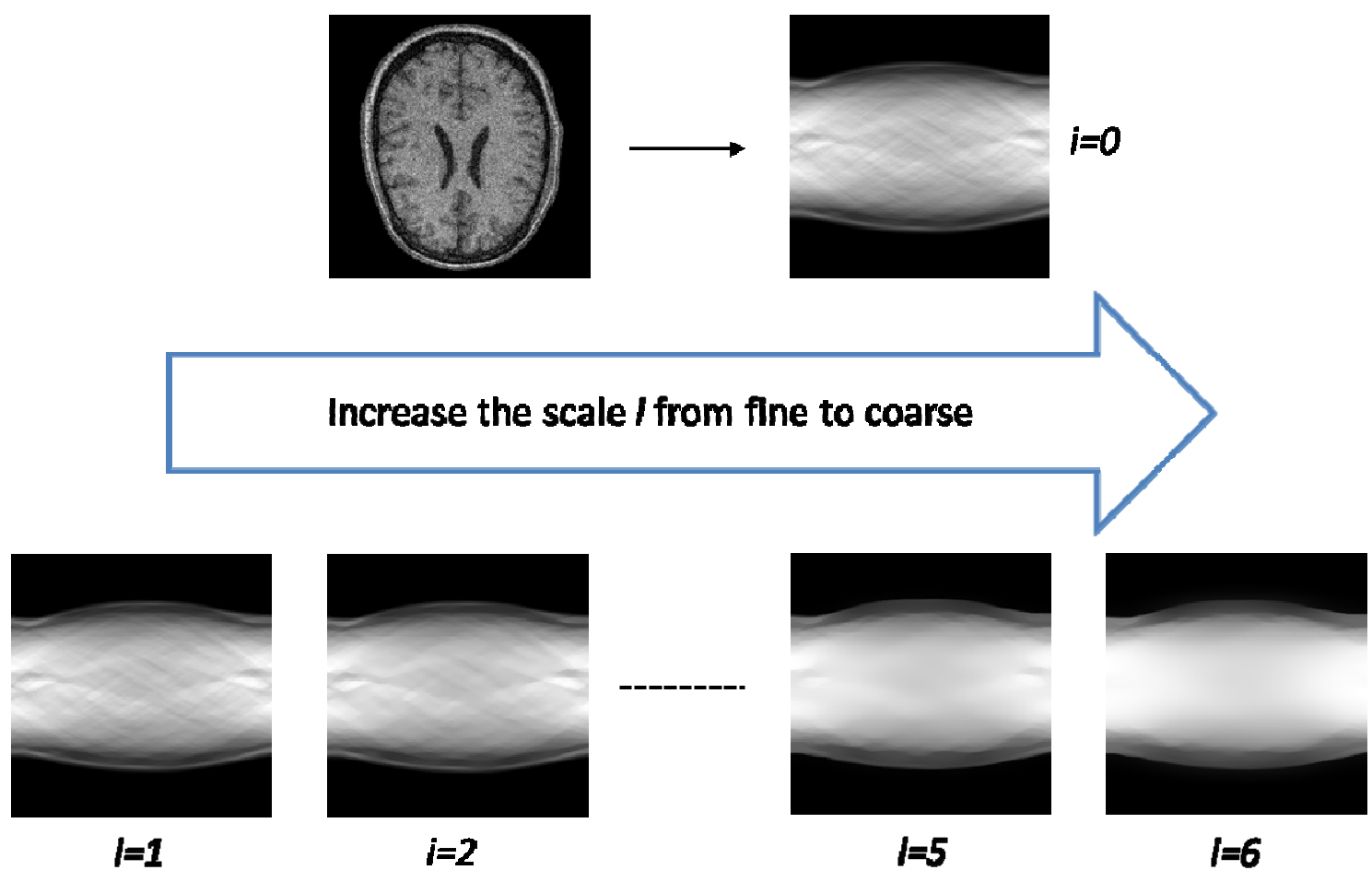

Figure 4. Scale-space constructed by bilateral filtering. The scale space is composed of a stack of the images filtered at different scales where $i=0$ is the original image. The greater the scale level, the less local information appears. 


\subsubsection{Gradient filtering and multiscale reconstruction}

For images at difference scales, we filter them in the vertical direction along the scales from the coarse to the fine levels using two sets of filters. The kernels are shown in Figure 5. We combine the upper half of the first filtered image with the lower half of the second filtered image in order to get a new sinogram. In the coarsest scale, the images are much smoothed and they only keep big edges. So for these two coarsest scales we can use a threshold or region growing method to get a mask. Figure 4 show that the 5th and 6th scales are greatly smoothed and that the big edges in both side still are preserved. The results from a coarse scale give a mask for the next fine scale and supervise the segmentation in the next fine scale. The reciprocal binary sinogram of the skull is obtained to reconstruct skull (Figure 6). The multiscale approach can effectively improve the segmentation speed and can avoid trapping into local solutions. Because of its multiscale bilateral filtering scheme, the method can be robust for noise MR images.
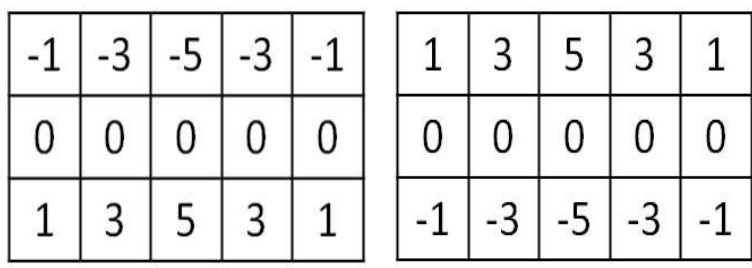

Figure 5. Kernels of the two filters

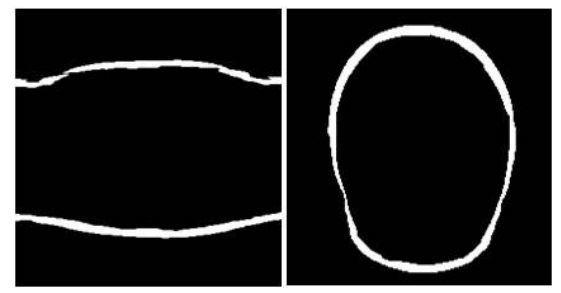

Figure 6. Segmented skull sinogram (left) and the reconstructed skull (right)

\section{SKULL RECONSTRUCTION}

After obtaining the reciprocal binary sinogram that includes only the skull, we perform reconstruction to get the skull image; and then in order to eliminate some artifacts introduced by reconstruction we take threshold to get real skull. We use filtered back projection (FBP) method for the reconstruction and it is described as

$$
f(x, y)=\int_{0}^{\pi} Q_{\theta}(x \cos \theta+y \sin \theta) d \theta
$$

With $Q_{\theta}$ is the ramp filtered projections.

\section{EXPERIMENT AND RESULTS}

In order to evaluate the performance of the segmentation method, the difference between the segmented images and the ground truth was computed using a variety of quantitative evaluation methods. We define six metrics to evaluate the segmentation results. The overlap ratios $\left(C_{1}, C_{2}\right.$, and $\left.C_{3}\right)$ and error ratios $\left(E_{1}, E_{2}\right.$, and $\left.E_{3}\right)$ were defined as follow.

$$
C_{1}=\frac{S_{1} \cap S_{2}}{S_{1} \cup S_{2}} \quad C_{2}=\frac{S_{1} \cap S_{2}}{S_{1}} \quad C_{3}=\frac{S_{2}}{S_{1} \cup S_{2}}
$$




$$
E_{1}=\frac{S_{1} \cup S_{2}-S_{1} \cap S_{2}}{S_{1} \cup S_{2}} \quad E_{2}=\frac{S_{1} \cup S_{2}-S_{1} \cap S_{2}}{S_{1}} \quad E_{3}=\frac{S_{1} \cup S_{2}-S_{1} \cap S_{2}}{S_{1} \cap S_{2}}
$$

Where $S_{1}$ is the ground truth and $S_{2}$ is the segmented result using our method, and here $S_{1}$ and $S_{2}$ both are binary images.

\subsection{Phantom data}

The method has been evaluated using brain phantom data, simulated MR images, and patient MRI and CT images. Figure 7 shows the result from the brain phantom images. In order to compare the results, we compute the absolute values of the difference between the segmented and real skull. As shown Figure 9, our approach is robust to additive noise. Even if we added 80\% noise and the result still is acceptable. According to Equations (9) and (10) we get the overlap ratios of more than $87.7 \pm 1.56 \%$ (Figure 8 ) and the error ratios of less than $13.9 \pm 2.79 \%$ for different noise levels (Figure 9). Figure 8 shows that even if the phantom is added $80 \%$ noise and the overlap ratios still exceed $85 \%$ and the error ratios are less than $17 \%$.
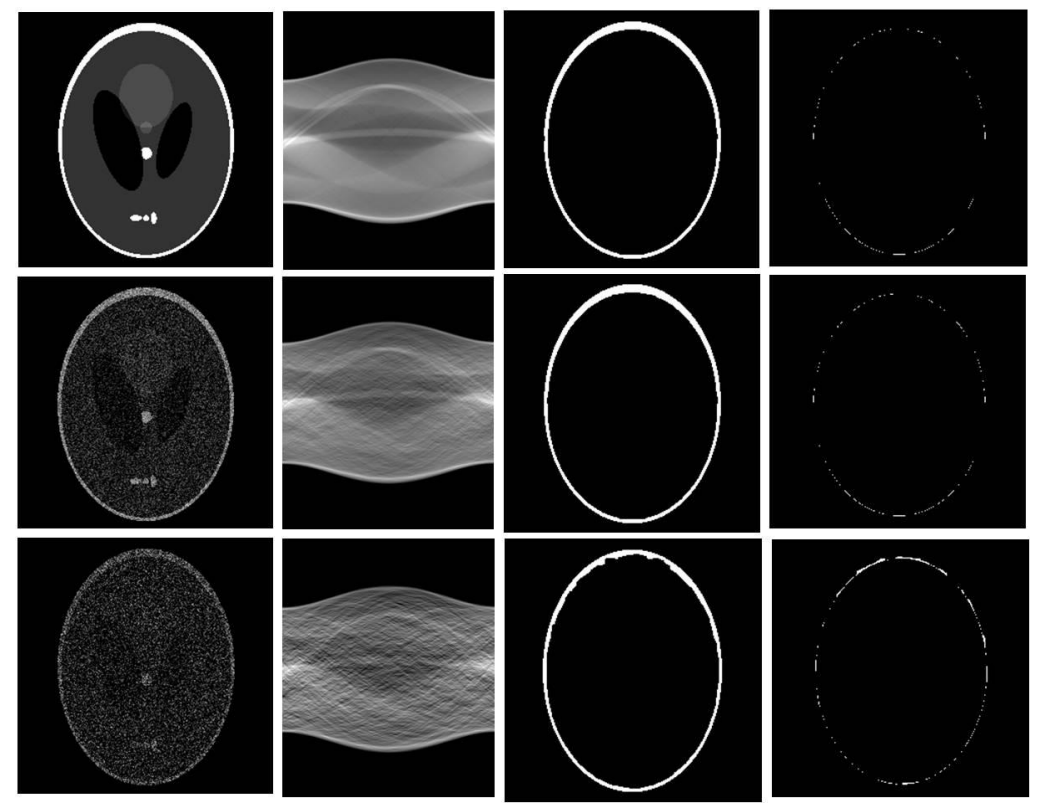

Figure 7. Segmentation results of the phantom with different noise levels. The first column from top to bottom shows the original phantom, and phantom with $30 \%$ and $80 \%$ white noise and with zero mean, respectively. The second column is the images after Radon transform. The third column is the reconstruction result (segmented skull). The fourth column is the difference between the segmented and real skull. 


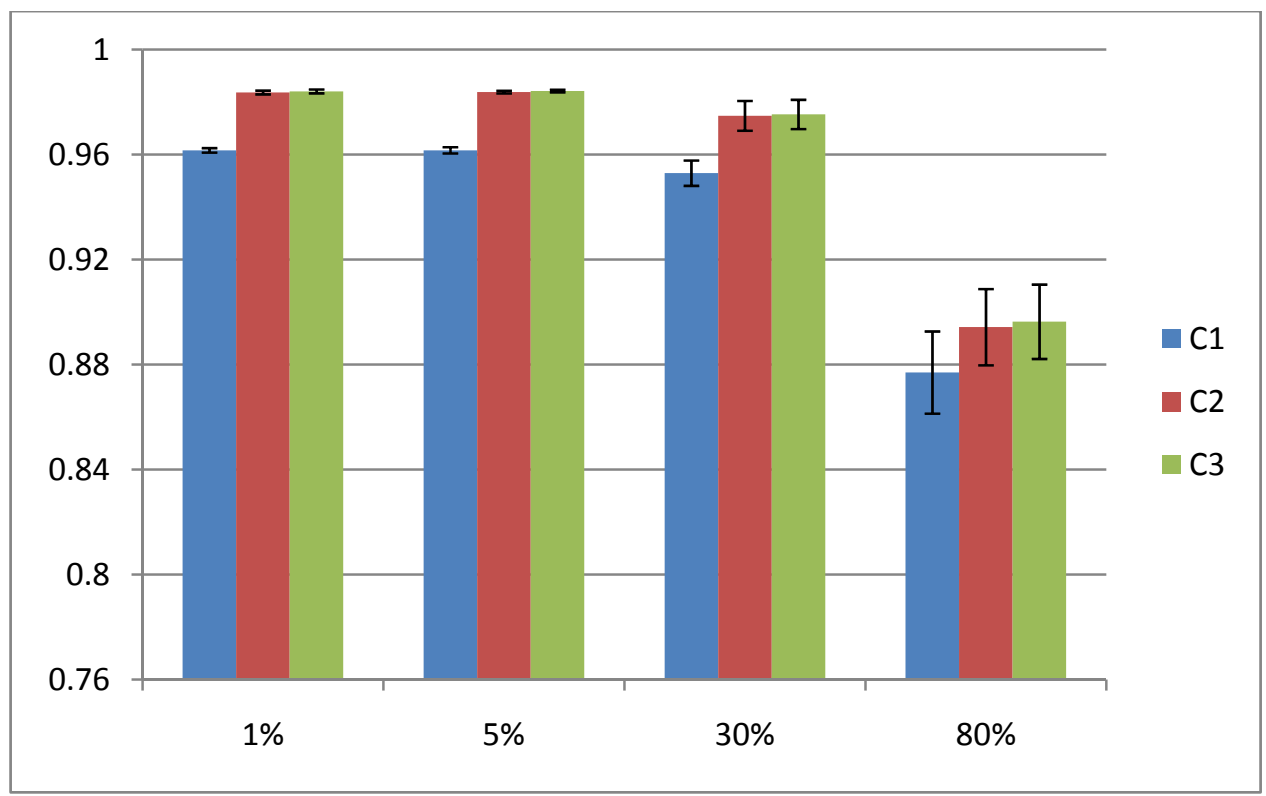

Figure 8. The overlap ratios of the segmentation at different noise levels

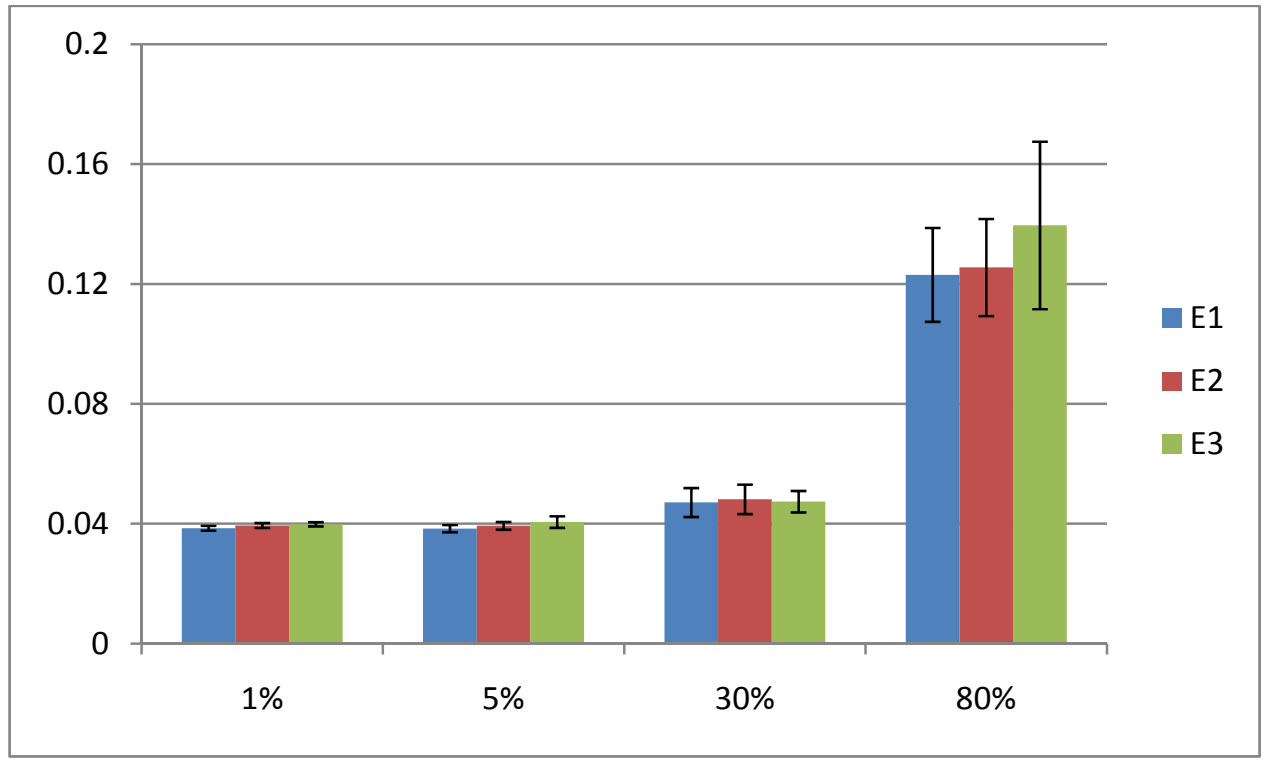

Figure 9. The error ratios of the segmentation at different noise levels

\subsection{Simulation data}

In Figure 10, we used the simulated brain data from PET-SORTEO Database [12]. Because the database provides the ground truth of the skull images, it can be used to evaluate our segmentation results. Ten sets of data from the database were used to perform statistical test and the results are shown in Table 1. The overlap ratios are more than $80 \%$ and the error ratios are less than $20 \%$ for all the segmentation. 

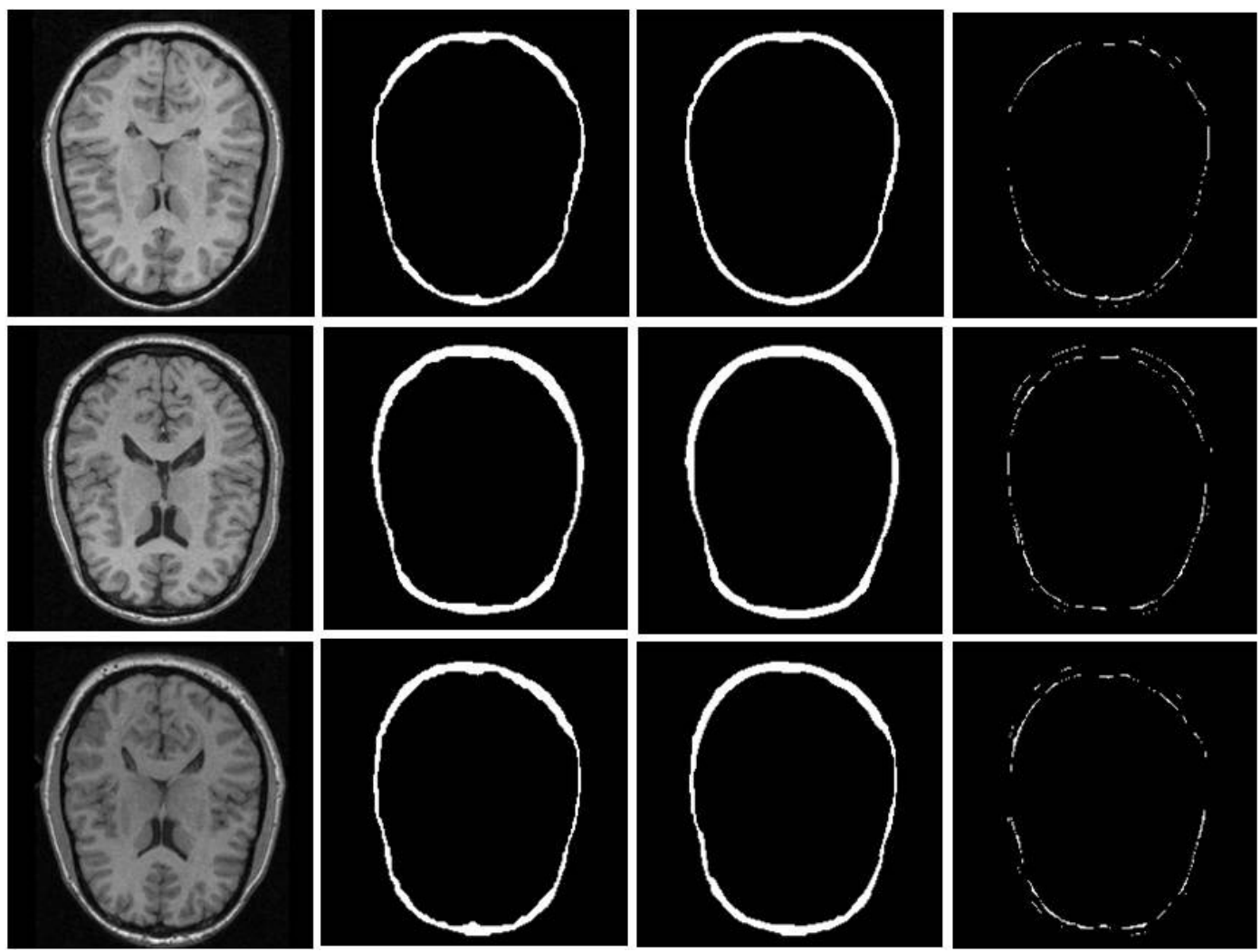

Figure 10. Segmention results of simulation brain MR images. The first column is the MR images at different locations. The second column is the corresponding skulls that the database offers. The third column is the segmented skull. The fourth column is difference between the segmented and "real" skull.

Table 1. Evaluation results for simulated brain data

\begin{tabular}{|c|c|c|c|c|c|c|}
\hline Name & $\mathrm{C} 1$ & $\mathrm{C} 2$ & $\mathrm{C} 3$ & $\mathrm{E} 1$ & $\mathrm{E} 2$ & $\mathrm{E} 3$ \\
\hline Average & 0.8537 & 0.9221 & 0.9289 & 0.1433 & 0.1529 & 0.1645 \\
\hline $\begin{array}{c}\text { Standard } \\
\text { Deviation }\end{array}$ & 0.0255 & 0.0275 & 0.0254 & 0.0213 & 0.0220 & 0.0246 \\
\hline
\end{tabular}

\subsection{Real MR and CT data}

In Figure 11, we applied our method to patient brain MRI data from the Vanderbilt Retrospective Registration Evaluation Dataset (RREP). First, we registered the CT to the corresponding MR images using our registration method [13] that has been evaluated in our laboratory. CT images provided the ground truth of the skull for the segmentation evaluation. The segmentation results from MR images are close to the results from CT images. 

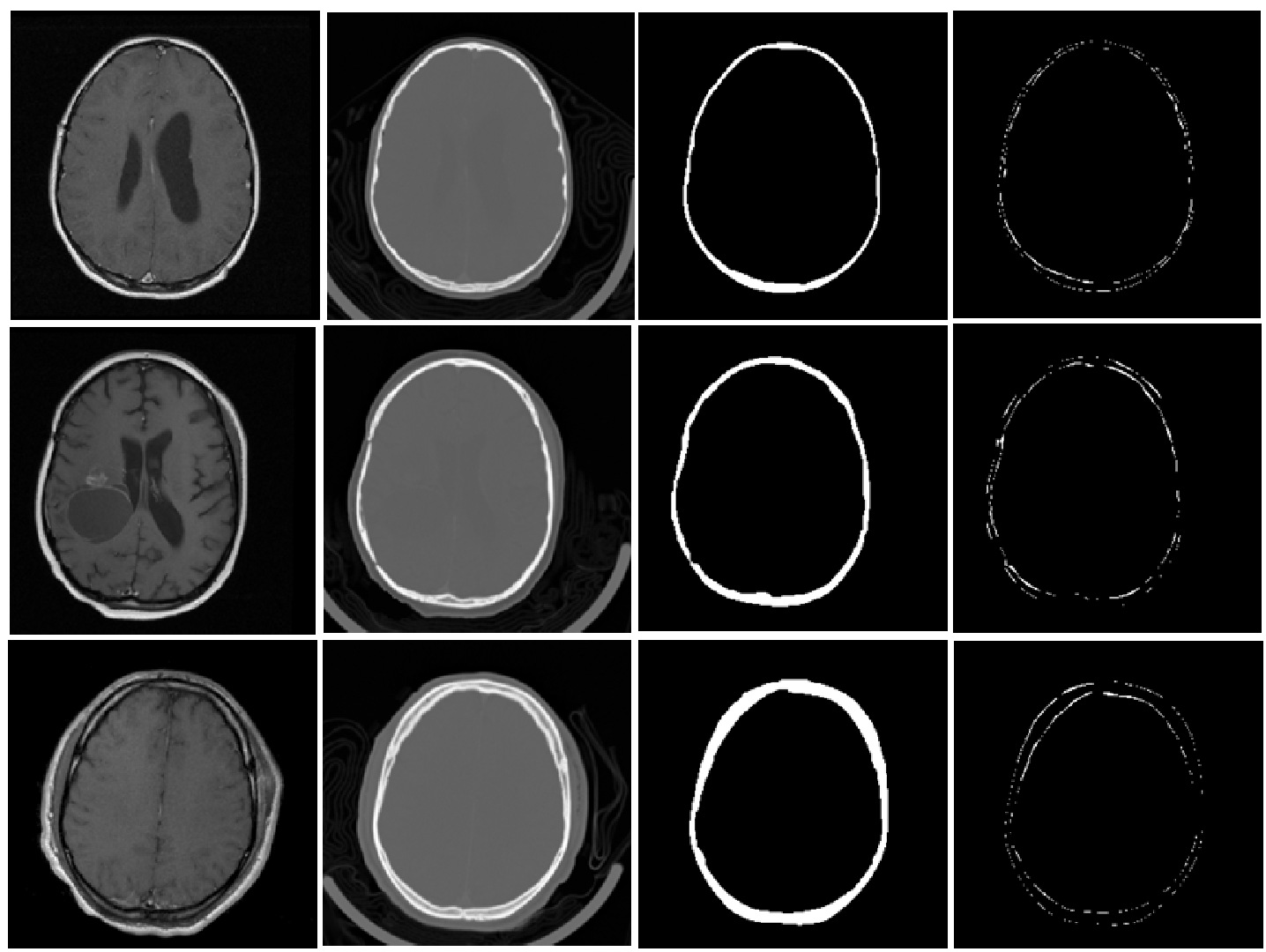

Figure 11. Segmentation results for real brain MRI and CT data. The first column is the MR images. The second column is the corresponding CT images. The third column is the segmented skull. And the fourth column is the corresponding difference between the segmented skull from MR images and those from CT.

\section{DISCUSSIONS AND CONCLUSIONS}

We developed and evaluated a multiscale skull segmentation method for brain MR images. We transform T1-weighted MR images to the Radon domain in order to detect the feature of the skull. In the Radon domain, we used a bilateral filter to effectively attenuate noise but preserve the edges. At same time we decompose images at multiple scales and increase the width of the spatial and range Gaussian by a factor of 2 at every scale. We use two kernels to filter all scale images except the coarsest one. We get a mask from the coarsest scale, which is used for the segmentation in the next scale. By this approach, we obtain accurate segmentation step-by-step and avoid being trapped into local minima. Our method is insensitive to random noise because we apply Radon transform and the bilateral filtering. Radon transform can improve the signal-to-noise level and the bilateral filtering keeps the edges and smoothes noise. Our method was evaluated using synthesized brain phantom image, a simulation brain MR database, and real MR images with CT images. The segmentation method is accurate and robust for noisy MR images with low contrast, which is useful for skull segmentation and subsequently for attenuation correction in combined PET/MRI applications. Our method can also be used in various neurological studies.

\section{ACKNOWLEDGEMENT}

This research is supported in part by the Georgia Cancer Coalition Distinguished Clinicians and Scientists Award (PI: Fei), by a pilot grant from the Atlanta Clinical Translational Science Institute (PHS grant: UL1 RR025008, KL2 RR025009 or TL1 RR025010), and by a pilot grant from the Emory Molecular and Translational Imaging Center (P50CA128301). 


\section{REFERENCES}

[1] Fei,B., Yang,X., and Wang,H., "An MRI-based attenuation correction method for combined PET/MRI applications," Proc. SPIE 2009.726208-726212 (2009).

[2] Segonne,F., Dale,A.M., Busa,E., Glessner,M., Salat,D., Hahn,H.K., and Fischl,B., "A hybrid approach to the skull stripping problem in MRI," NeuroImage.221060-1075 (2004).

[3] Zhuang,A.H., Valentino,D.J., and Toga,A.W., "Skull-stripping magnetic resonance brain images using a modelbased level set," NeuroImage.3279-92 (2006).

[4] Soltanian-Zadeh,H. and Windham,J.P., "A multiresolution approach for contour extraction from brain images," Med. Phys.241844-1853 (1997).

[5] Robson,M.D. and Bydder,G.M., "Clinical ultrashort echo time imaging of bone and other connective tissues," NMR Biomed.19765-780 (2006).

[6] Tyler,D.J., Robson,M.D., Henkelman,R.M., Young,I.R., and Bydder,G.M., "Magnetic resonance imaging with ultrashort TE (UTE) PULSE sequences: technical considerations," J. Magn Reson. Imaging.25279-289 (2007).

[7] Fennema-Notestine,C., Ozyurt,I.B., Clark,C.P., Morris,S., Bischoff-Grethe,A., Bondi,M.W., Jernigan,T.L., Fischl,B., Segonne,F., Shattuck,D.W., Leahy,R.M., Rex,D.E., Toga,A.W., Zou,K.H., and Brown,G.G., "Quantitative evaluation of automated skull-stripping methods applied to contemporary and legacy images: effects of diagnosis, bias correction, and slice location," Hum. Brain Mapp.2799-113 (2006).

[8] Boesen,K., Rehm,K., Schaper,K., Stoltzner,S., Woods,R., Luders,E., and Rottenberg,D., "Quantitative comparison of four brain extraction algorithms," NeuroImage.221255-1261 (2004).

[9] Rehm,K., Schaper,K., Anderson,J., Woods,R., Stoltzner,S., and Rottenberg,D., "Putting our heads together: a consensus approach to brain/non-brain segmentation in T1-weighted MR volumes," NeuroImage.22(3), 12621270 (2004).

[10] Tomasi,C. and Manduchi,R., "Bilateral filtering for gray and color images," 839-846 (1998).

[11] Fattal,R., Agrawala,M., and Rusinkiewicz,S., "Multiscale shape and detail enhancement from multi-light image collections," Acm Transactions on Graphics.26(3), (2007).

[12] Reilhac,A., Lartizien,C., Costes,N., Sans,S., Comtat,C., Gunn,R.N., and Evans,A.C., "PET-SORTEO: a Monte Carlo-based Simulator with high count rate capabilities," Nuclear Science, IEEE Transactions on.51(1), 46-52 (2004).

[13] Fei,B., Wang,H., Muzic,R.F., Jr., Flask,C., Wilson,D.L., Duerk,J.L., Feyes,D.K., and Oleinick,N.L., "Deformable and rigid registration of MRI and microPET images for photodynamic therapy of cancer in mice," Med. Phys.33753-760 (2006). 